\title{
A Survey of Brain-Computer-Interaction Methods and Algorithms
}

\author{
Preeti Ghasad $^{1}$, V.N. Sahare ${ }^{2}$ \\ Dept., of Computer Science and Engg., G.H Raisoni Institute of Engg., and Technology for Women, Nagpur, Maharashtra, India ${ }^{1}$ \\ Assistant Professor, Dept., of Computer Science and Engg., G.H Raisoni Institute of Engg., and Technology for Women Nagpur,
}

\section{Maharashtra, India ${ }^{2}$}

\begin{abstract}
In recent years several researchers are practicing on multiple body-machine interfacing techniques. Many techniques were successfully implemented and launched as a commercial products for medical and bioinformatics communications. Still concerning electro-oculography interfacing for Human Computer Interface (HCI) has wide scope of development and real life implementations. Like EEG, EMG and ECG, EOG doesn't provide important body parameters which could be used for disease diagnosis but it has very wide applications of machine interactions. The eye movement is known to be a essential communication tool for any person hence EOG could be used by Paralyzed stroke patients are unable to normally communicate with their environment. These patients can control the only part of their body is their eyeballs. Proposed system will identify the variations in electric signal strength through voltage level near the eye area and generates a signal in order to control the multimode interactive device. Different type of instrumental amplifiers could be used for better results and interfaced with communication devices.
\end{abstract}

Keywords: Human Computer Interface (HCI), Electrooculogram (EOG), Electroencephalograph (EEG), Electrocardiogram $(E C G)$, Electromyogram $(E M G)$.

\section{INTRODUCTION}

BRAIN computer interface (BCI), often called as a mindmachine interface (MMI), or sometimes also known as straight neural interface or a brain-machine interface (BMI), it is a direct communication pathway between the brain and an external device. BCIs are frequently anticipated for support, augmenting, or repairing human cognitive or sensory functions. A braincomputer interface (BCI) is a device that enables severely disabled people to communicate and interact with their environments using their brain waves. Mostly researchers have investigated BCI in humans using scalp-recorded electroencephalography or intracranial electrocorticography. The use of brain signals which obtained directly from stereotactic depth electrodes to control a BCI has not previously been explored. Most researchers are investigating BCI in humans has used scalp-recorded electroencephalography or intracranial electroorticography.In other methodology; most famous approaches involve the use of a camera to visually track the eye. However, this method has problems that the eyes of user must always be open. Proposed systems will detect the variations in electric signal strength through the voltage level near the eye area and generates a wireless radio frequency signals in order to control the robotic model. By implementing this system we can further extend it to bio enabled human body parts to control through brain waves.

Electroencephalography (EEG) is the most studied potential non-invasive interface occurs mainly due to its fine temporal declaration, easy to use, high portability and low cost set-up. But as well as the technology's susceptibility to noise, another considerable difficulty to using EEG as a brain-computer interface is that the

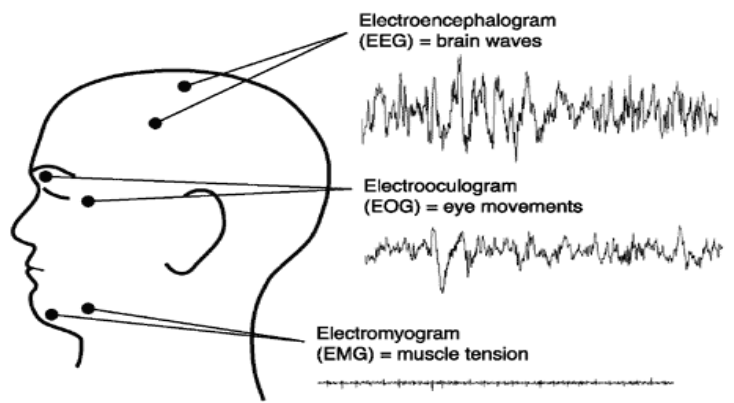

Fig1. EEG, EOG, EMG

extensive training is required before users can work on this technology.

For example, in these experiments several paralyzed people are trained to self-regulate the slow cortical potentials in their EEG to such an extent that these signals could be used only as a binary signal to control a computer cursor. By controlling their brain waves, the experiment saw the ten patients trained to move a computer cursor. The process was slow, it took more than an hour for patients to write 100 characters with the cursor, while training often required many months.

\section{RELATED WORK}

MINGMIN YAN SOU GO, HIROKI TAMURA,KOICHI TANNO [1], proposed the mouse cursor control system for ALS patients using EOG and EEG signals. They introduced the algorithm using alternating current and direct current of EOG corresponding to the drift. They also took measurement to examine whether the subject could control their eye movement consciously. The EEG signals were not used to control the mouse movement, but to determine the subject's control state. 
Takamitsu Matsubara [2], proposed a multiuser myoelectric interface that can easily adapt to novel users. When a user performs different motions different electromyography (EMG) signals are measured.

$\mathrm{Xin} \mathrm{Yi}$, Jun Jia [3], proposed a system which achieves restoration of synchronized blink through processing the myoelectric signal of orbicularis oculi at the normal side in real-time as the trigger to stimulate the paralyzed eyelid. Joshua Michael Peschel [4], this paper surveys the human machine interaction technologies supporting the Mission Specialist role in unmanned aerial systems (UASs).

Palumbo1, 2, P. Vizza1, P. Veltri1 [5], proposed the design of an amplifier for EEG signals. The knowledge of EEG signals is very important for the detection of cerebral rhythmic and any abnormalities. Brain waves can be classified with the human activity; these signals represent an extremely low dynamic range $(5-200 \mu \mathrm{V})$, having a frequency band of $0.5-40 \mathrm{~Hz}$. Brain electrical potentials are acquired by using the special surface electrodes, by mounting it monopolarly: sensing electrode is placed above the skull of interest, while along the mid line a second electrode is positioned for reference electrode.

Andre's U' beda, Eduardo Ia'ñez, and Jose' M. Azor'in [6], proposed a new portable and wireless interface based on electrooculography (EOG) aimed at people with severe motor disorders. This interface allows us detecting the movement of the eyes measuring the potential between the cornea and the retina. In order to register this potential the interface uses five electrodes placed around the eyes of the user. In order to detect the movement of the eyes a processing algorithm of the EOG signals has been developed. In comparison to commercial devices this interface has many advantages. It is a low cost and small sized device having USB compatibility. As it works with batteries and USB supply it does not require power supply from the network. In addition, this interface has been used to control a real robot arm. The accuracy and time required have been measured showing that the user is capable of controlling the robot using only his/her eyes with satisfactory results.

\section{PROPOSED SYSTEM}

In this paper proposes a new methodology present electrooculogram (EOG) signals that can be used for human computer interface efficiently. The main objective of the system is to detect the electrical signal near eye area and using electrodes system which will try to identify the changes in electric pulse in order to conclude the motion to be taken.

The working of the proposed system is explained below:

A. Module 1- Designing a wireless robotic vehicle

It is a hardware model which includes the wireless robotic vehicle, in four different directions it can be controlled. User can have access to this vehicle using radio frequency enabled circuitry through brain signals generated using eye motion.

B. Module 2- Designing a computer cursor and application control

Likewise user can control the computer cursor and the applications using electrical signals. This will enable disabled patients to have good access over computer system. In order to implement this there will be a microcontroller to USB interfacing circuitry which will convert microcontroller signals into computer understandable signals, which will then get processed by software program.

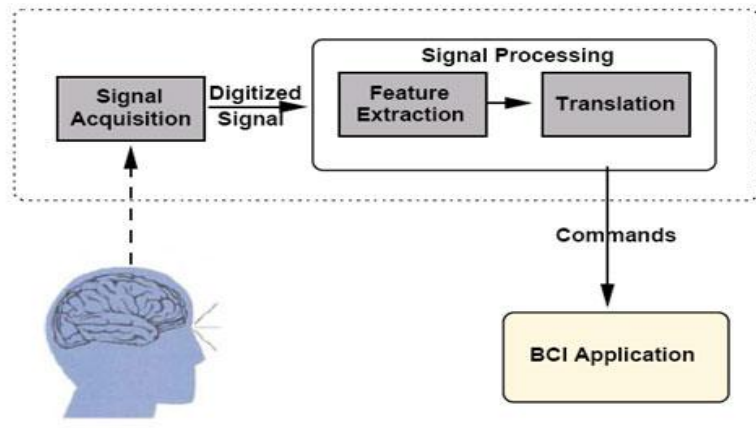

Fig2.Phases in Brain-Computer Interfaces

Figure 3 gives the illustration of the system architecture of the proposed system below:

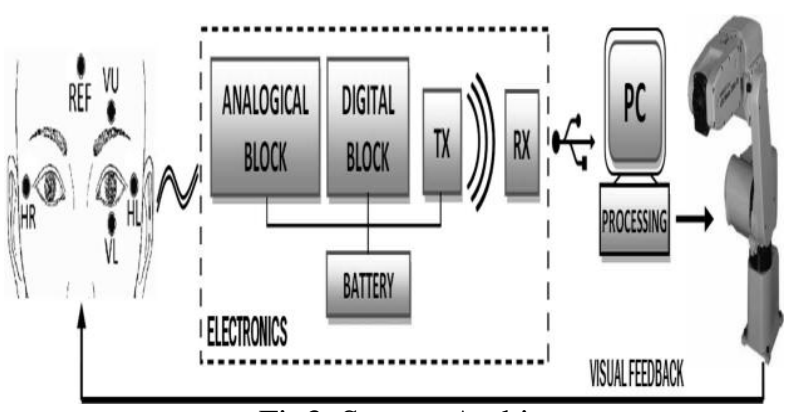

Fig3. System Architecture

In this we are designing an embedded system, which will convert the electrical signal near eye area by using electrodes system,into digital signals. The signals we are getting will be in analog form so this signals are converted into digital form with the help of embedded system for further interface.

\section{IV.CONCLUSION}

The proposed system will detect the variations in electrical signal strength through voltage level near the eye area and generates a wireless radio frequency signals in order to control the robotic prototype model. The proposed system, allows physically paralyzed patient to communicate and control computer and hardware system using brain waves electrical signals by using eyeballs. The proposed system can be further extended to bio enabled human body parts to control through brain waves.

\section{REFERENCES}

[1] Mingmin Yan,"Communication system using EOG for persons with disabilities and its judgment by EEG",Springer on Artif Life Robotics 2014,DOI 10.1007s.

[2] Takamitsu Matsubara, "Bilinear Modeling of EMG Signals to Extract User-Independent Features for Multiuser Myoelectric Interface",IEEE Transactions on Biomedical Engineering, Vol. 60, No. 8, August 2013.

[3] Xin Yi, "A Blink Restoration System With Contralateral EMG Triggered Stimulation and Real-Time Artifact Blanking",IEEE Transactions on Biomedical Circuits asnd Systems, Vol. 7, No. 2, April 2013. 
International Journal of Advanced Research in Computer and Communication Engineering Vol. 4, Issue 1, January 2015

[4] Joshua Michael Peschel, "On the Human-Machine Interaction of Unmanned Aerial System Mission Specialists", IEEE Transactions on Human-machine Systems, Vol. 43, No. 1, January 2013.

[5] Andre's U' beda, Eduardo Ia ñez, and Jose' M. Azor'in,"Wireless and Portable EOG-Based Interface for Assisting Disabled People", IEEE Transactions on Mechatronics, Vol. 16, No. 5, October 2011.

[6] Palumbo1,2, P. Vizza1, P. Veltri1,“ Design Of An Electronic Device For Brain Computer Interface Applications", International

\section{BIOGRAPHY}

Preeti Ghasad, Masters in Wireless

Communication and Computing. 